\title{
The Usefulness of Renal Doppler Parameters in Chronic Kidney Disease: Is There a Cut-Off Value to Estimate End Stage Kidney Disease?
}

\author{
Bozkurt Gulek ${ }^{*}$, Gokhan Soker ${ }^{1}$, Ertugrul Erken' ${ }^{2}$, Fatma Ulku Adam ${ }^{3}$, Halil Ibrahim Varan ${ }^{3}$, \\ Sibel Ada ${ }^{3}$, Nazan Z. Alparslan ${ }^{4}$, Omer Kaya ${ }^{1}$, Eren Erken ${ }^{5}$, Behice Durgun 6 \\ ${ }^{1}$ Department of Radiology, Numune Teaching and Research Hospital, Adana, Turkey \\ ${ }^{2}$ Department of Nephrology, Faculty of Medicine, Gaziosmanpaşa University, Tokat, Turkey \\ ${ }^{3}$ Department of Nephrology, Numune Teaching and Research Hospital, Adana, Turkey \\ ${ }^{4}$ Department of Biostatistics, Faculty of Medicine, Cukurova University, Adana, Turkey \\ ${ }^{5}$ Department of Rheumatology-Immunology, Faculty of Medicine, Cukurova University, Adana, Turkey \\ ${ }^{6}$ Department of Anatomy, Faculty of Medicine, Cukurova University, Adana, Turkey \\ Email: "bozkurtgulek@gmail.com
}

Received 24 December 2015; accepted 8 March 2016; published 11 March 2016

Copyright (C) 2016 by authors and Scientific Research Publishing Inc.

This work is licensed under the Creative Commons Attribution International License (CC BY).

http://creativecommons.org/licenses/by/4.0/

c) (i) Open Access

\begin{abstract}
Aims: In this study, Doppler parameters were studied in patients with advanced stage renal disease, and the relationship between Doppler parameters and renal disease stage was investigated. Doppler values were evaluated for a cut-off value between normal and end-stage kidney disease group. Materials and methods: 50 patients with chronic kidney disease and 15 patients belonging to the same age group and with normal serum creatinine levels were comprised of the study and control groups. Resistivity and pulsatality indices were measured at the main renal arteries and interlobular arteries at both sides. Results: Statistically significant differences were found between the PI and RI values obtained from the main renal and interlobular arteries. PI sums were compared with those from the control group, and a cut-off value of 2.15 was found, with a sensitivity of $90 \%$ and a specificity of 86.7\%. Conclusion: Any increase in the RI and PI values obtained from the main renal and interlobular arteries must bring to mind the possibility of advancing renal damage and interstitial fibrosis. Patients with and without renal parenchymal damage can be differentiated by means of comparing the total PI values obtained from the right and left main renal and interlobular arteries.
\end{abstract}

\section{Keywords}

Ultrasonography, Doppler, Renal Insufficiency, Chronic

\footnotetext{
${ }^{*}$ Corresponding author.
}

How to cite this paper: Gulek, B., Soker, G., Erken, E., Adam, F.U., Varan, H.I., Ada, S., Alparslan, N.Z., Kaya, O., Erken, E. and Durgun, B. (2016) The Usefulness of Renal Doppler Parameters in Chronic Kidney Disease: Is There a Cut-Off Value to Estimate End Stage Kidney Disease? Open Journal of Radiology, 6, 18-23. http://dx.doi.org/10.4236/ojrad.2016.61003 


\section{Introduction}

Chronic kidney disease is in the course of becoming a bigger major public health issue [1] [2]. In very few of these patients, very rapid impairment of renal function takes place, and even fewer patients can reach the terminal renal disease phase [3] [4]. It is of vital importance to predict the late term prognosis of these patients. Some of these predictors are arterial hypertension, proteinuria, and baseline renal function [5]. Interstitial fibrosis also supplies valuable predictive data about prognosis. On the other hand, Doppler ultrasonography (US) gives very important and valuable data about both the renal morphology and vasculature [6] [7]. The renal intraparenchymal arterial flow pattern is influenced by structural and functional factors, and the flow wave is created by vascular compliance and resistance. This is why pathological conditions such as interstitial fibrosis have a strong impact on the indices obtained from Doppler examinations [8]. Recent studies have shown that there is a close relationship between Doppler parameters (especially the resistive index) and tubulointerstitial and vascular damage [6]. In this study, intra and extrarenal arterial flow Doppler parameters were studied in patients with advanced stage renal disease, and the relationship between Doppler parameters and renal disease stage was investigated.

\section{Materials and Methods}

The protocol of this prospective study was approved by the Ethical Committee of our hospital, which is a State Training and Research Health Center. The study was conducted in accordance with the Declaration of Helsinki. All patients gave their full informed consents prior to the study. The study group comprised 50 patients. These were advanced-stage renal disease patients with persistent proteinuria and persistently high-levels of serum creatinine. Proteinuria was defined as a urinary protein level of over $0.3 \mathrm{gm} / \mathrm{gm}$ creatinine, whereas a high level of serum creatinine was determined more than $1.08 \mathrm{mg} / \mathrm{dl}$ for men and $0.84 \mathrm{mg} / \mathrm{dl}$ for women. Patients with renal artery stenosis (RAS), acute renal failure, and hepatorenal syndrome, were excluded from the study. The patients were categorized as being Stage 1 - 5, according to their glomerular filtration rates (GFR). These categorizations were as follows: GFH $>90 \mathrm{ml} / \mathrm{min} / 1.73 \mathrm{~m}^{2}$ was Stage $1 ; 60-89 \mathrm{ml} / \mathrm{min} / 1.73 \mathrm{~m}^{2}$ was Stage 2; 30 - 59 $\mathrm{ml} / \mathrm{min} / 1.73 \mathrm{~m}^{2}$ was Stage 3, $15-29 \mathrm{ml} / \mathrm{min} / 1.73 \mathrm{~m}^{2}$ was Stage 4 ; and $<15 \mathrm{ml} / \mathrm{min} / 1.73 \mathrm{~m}^{2}$ was Stage 5 . GFR was calculated according to the Modification of Diet in Renal Disease (MDRD) formula [9]. All patients were examined by B-Mode and Doppler US in the lateral decubitus position, following a fasting period of at least 6 hours. US and Doppler US examinations were performed by a single experienced radiologist, by the utilization of a $3.5 \mathrm{MHz}$ abdominal convex transducer of a Mindray DC-7 machine. The kidneys were first assessed morphologically. The longest axes and the mean parenchymal thicknesses of both of the kidneys were measured and recorded. Increase in renal parenchymal echogenicity was assessed and recorded as Grade 1, 2 and 3, as being light, moderate, and advanced, in sequence order. Then, Doppler flow samples were obtained from the proximal or median segments of the right and left main renal arteries, utilizing a Doppler angle of $30^{\circ}-60^{\circ}$. Maximum systolic (RA PSV) and end-diastolic (RA EDV) flow velocities were measured from the renal arteries. The resistivity index (RI) value from the renal arteries was assessed according to the RA PSV-RA EDV/RA PSV formula [10]. The pulsatility index (PI) value from the renal arteries was determined according to the RA PSV-RA EDV/Mean Renal Arterial Flow Velocity formula [11]. All measurements were made at least twice, and the mean result was recorded as the final value. Following all these procedures, the same measurements were made at interlobular arteries situated at the upper, middle, and lower portions of the kidneys. Again, the mean results were recorded as the final values. 15 patients belonging to the same age group and with normal serum creatinine levels and no proteinuria comprised the control group. The PI values obtained from the right and left renal arteries were added and their sum was recorded. On the other hand, those PI values drawn from the right and left interlobular arteries, too, were added and their sum was recorded. Then, these sums were compared with those from the control group.

As for the analysis of the data, the test was used for the comparison of the patient and control groups, whereas the Variance Analysis (ANOVA) test was utilized for the evaluation of the results obtained from different renal disease stages. When the $\mathrm{P}$ value was lower than 0.05 , the results were accepted as statistically significant. The RI and PI values obtained from the two groups were evaluated for a cut-off value by the administration of the Receiver Operating Characteristics (ROC) curve analysis method.

\section{Results}

50 patients possessing the inclusion criteriae were included in the study. 15 patients comprised the control group. 
There were 28 males (56) and 22 females (44\%) in the study group, whereas the control group consisted of 8 males (53\%) and 7 females (47\%). There were no statistically significant differences between the patient and control groups in terms of gender. The mean age was $61.3 \pm 12.3$ years old in the study, and $52.7 \pm 14.2$ years old in the control, groups. No statistically significant difference was found between the two groups in terms of age. In 5 patients, Doppler measurements could not be made from unilateral intrarenal arteries, due to gas artefacts and inability to hold breath. In 1 patient on the other hand, measurements could not be processed from both sides. The mean RI value from the right main renal artery was $0.72 \pm 0.07$ in the patient group, while it was found to be $0.64 \pm 0.04$ in the control group. The mean PI value of the right main renal artery was $1.46 \pm 0.36$ in the study group, whereas it was $1.05 \pm 0.24$ in the control group. The mean RI value from the left main renal artery was $0.73 \pm 0.077$ in the study group, while it was $0.65 \pm 0.05$ in the control group. The mean PI values sampled from the left main artery were found to be $1.61 \pm 0.42$ and $0.99 \pm 0.14$, in the study and control groups, in sequence order. Statistically significant differences were found between the PI and RI values obtained from the right and left main renal arteries. The mean RI value obtained from the interlobular arteries was found to be $0.68 \pm 0.074$ in the patient group, whereas it was $0.64 \pm 0.036$ in the control group. The mean PI values sampled from interlobular arteries were found to be $1.26 \pm 0.31$ and $1.02 \pm 0.16$, for the patient and control groups, consecutively. Even though the mean RI samplings made from the interlobular arteries showed some difference between the two groups, this difference was not statistically significant. On the other hand, a statistically significant difference was found between the PI values of the two groups. The mean RI values obtained from the left interlobular arteries were $0.69 \pm 0.06$ for the study, and $0.64 \pm 0.03$ for the control, groups.

The PI values, on the other hand, were found as $1.28 \pm 0.25$ and $1.06 \pm 0.12$, for the two groups, consecutively. Statistically significant differences were found between the patient and control groups in terms of the RI and PI values obtained from the left renal interlobular arteries (Figure 1 and Figure 2). The mean long axis diameter of the right kidney was $94.9 \pm 15.9 \mathrm{~mm}$ in the study, and $108.5 \pm 10.7 \mathrm{~mm}$ in the control, groups. The mean parenchymal thickness for the right kidney was measured as $10.01 \pm 2.63 \mathrm{~mm}$ in the study group, while it was 13.3 $\pm 1.36 \mathrm{~mm}$ in the control group. The mean left renal longitudinal axis measurement was $97.84 \pm 14.3 \mathrm{~mm}$ for the study, and $111.6 \pm 8.09 \mathrm{~mm}$ for the control, groups, consecutively. The mean left renal parenchymal thickness measurements on the other hand, were found to be $11.3 \pm 3.02 \mathrm{~mm}$ for the study, and $12.61 \pm 17.76 \mathrm{~mm}$ for the

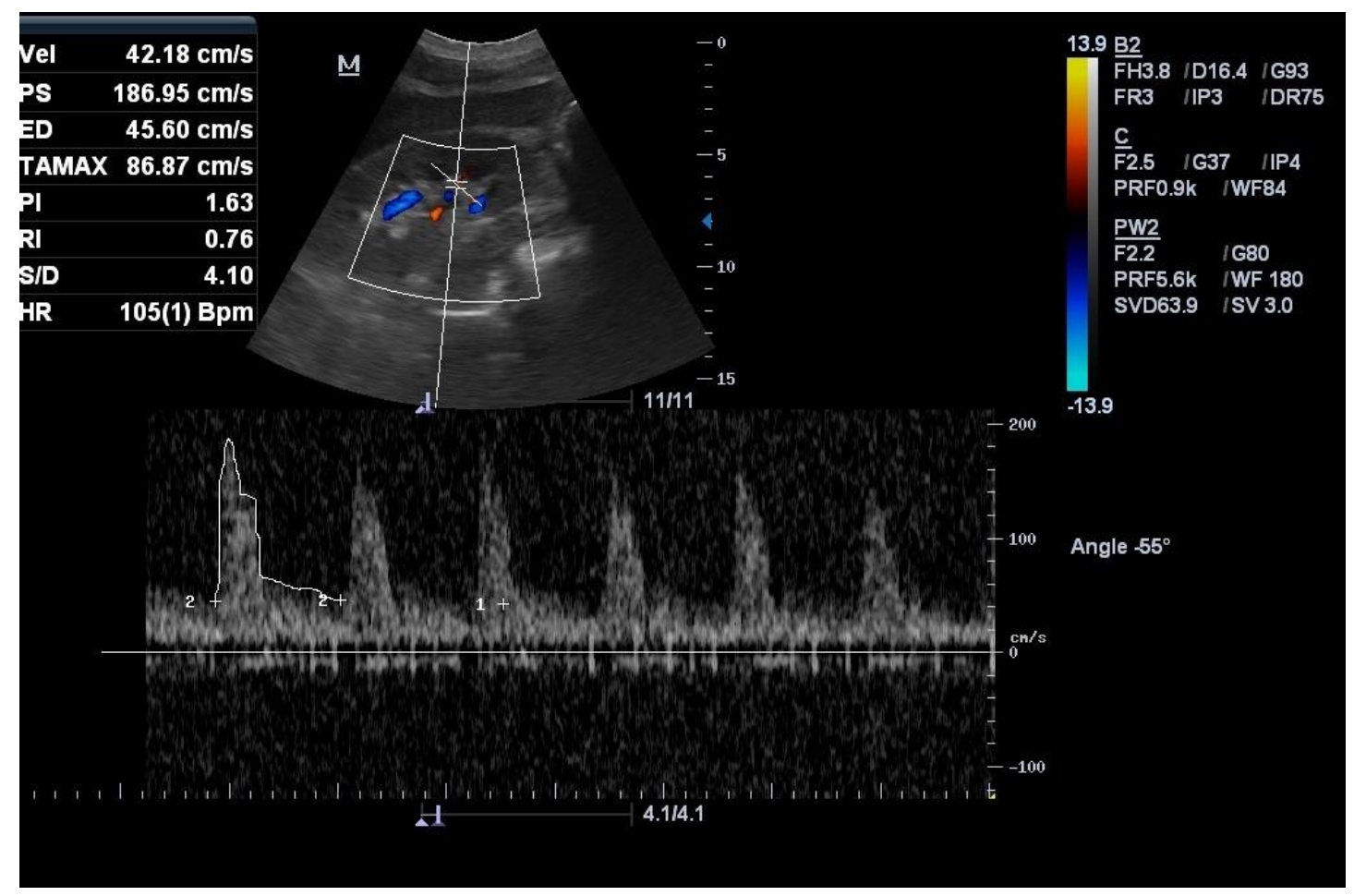

Figure 1. Doppler sonograms and spectral waveforms of a 56-year-old woman with chronic kidney disease show elevated RI and PI values. 


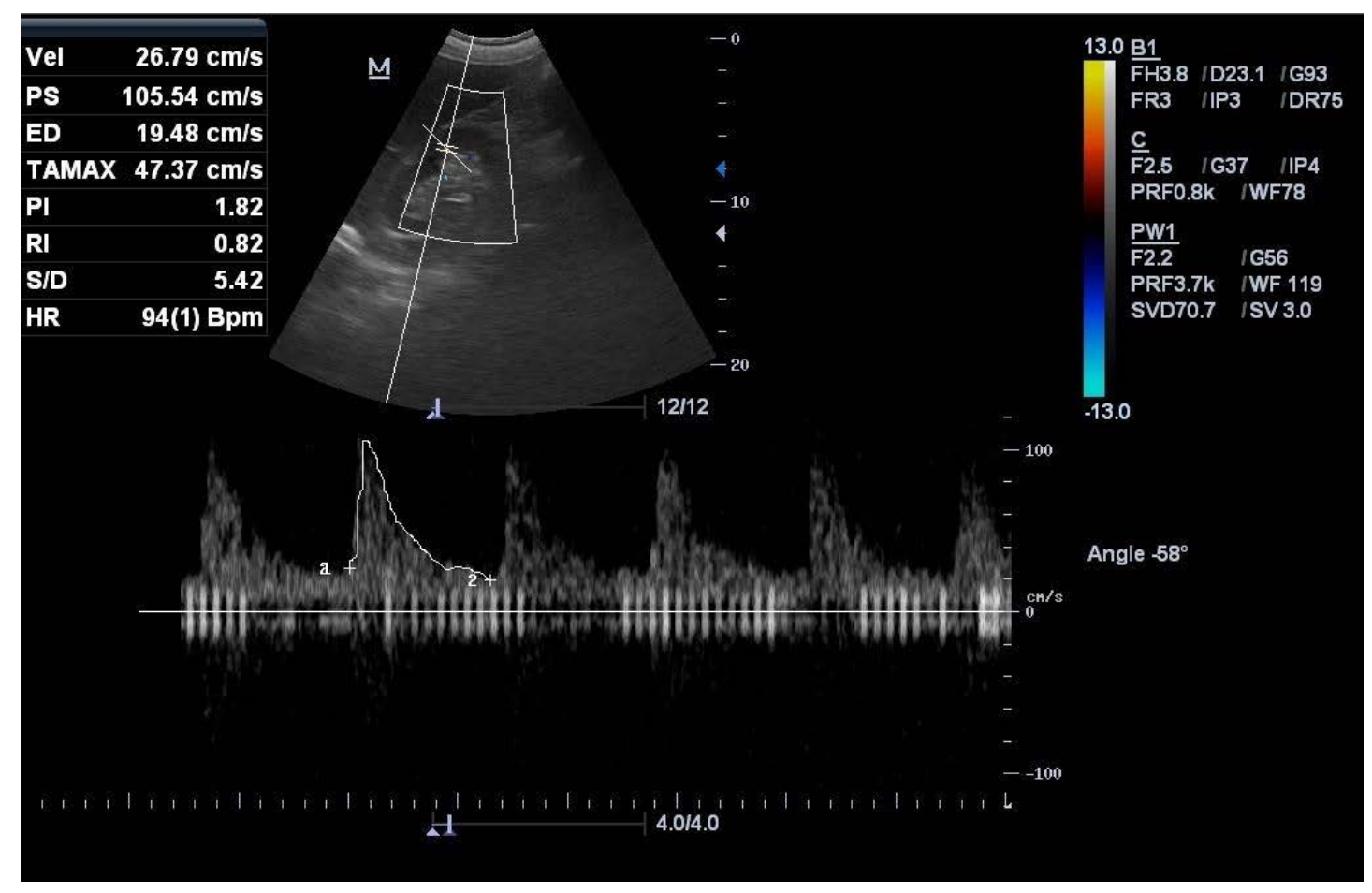

Figure 2. Doppler sonograms and spectral waveforms of a 69-year-old man with chronic kidney disease. Intrarenal artery shows elevated RI and PI values.

control, groups, in order. Statistically significant differences were found between the study and control groups in terms of mean renal dimensions. While statistically significant differences were found between the study and control groups in terms of right renal parenchymal thicknesses, the same measurements obtained from the left kidneys did not demonstrate any significant differences.

Another striking finding was the presence of statistically significant differences between the renal disease stages in the patient group, in terms of all parameters studied.

The results drawn by adding together all of the PI values obtained from right and left renal main or interlobar arteries were also compared between the patient and control groups. Resultant PI values were obtained by adding together the PI values from the main renal arteries. The same procedure was done for the right and left interlobular arteries, too. Then, these resultant PI sums were compared with those from the control group, and a cut-off value of 2.15 was found, with a sensitivity of $90 \%$ and a specificity of $86.7 \%$.

\section{Discussion}

Chronic renal disease is a risk factor for terminal stage renal disease and cardiovascular diseases. Early diagnosis and proper medical treatment is essential for the prevention of these diseases. It is of vital importance to evaluate the renal functions and predict the long-term outcomes. The presence of interstitial fibrosis and loss of capillaries and tubules are a general result of progressive renal disease, and GFR loss, on the other hand, is closely related with renal functions and long-term prognosis [12]. The relations between RI and pathological conditions such as glomerulosclerosis and tubulointerstitial and vascular damage have been reported in previous studies [13]-[15]. In many other studies, the relations between renal Doppler parameters and conditions such as renal functions and histologic damage have been investigated. Izumi et al. have reported that the differential diagnosis of acute tubular necrosis and prerenal azotemia may be made by means of the renal resistivity index [16]. Sugiura and Wada have reported at the end of their study that interstitial injury may be evaluated by studying the atrophic index and RI [17]. Boddi et al. have emphasized the value of RI in the early diagnosis of chronic tubulointerstitial nephritis. In our study too, statistically significant differences were found between the control group and stages 2 - 4 chronic renal disease patients, in terms of the RI values measured from the main renal and interlobar arteries. Even though statistically significant differences were detected between the two groups con- 
cerning the PI values measured from the intrarenal arterial branches, no statistically significant difference could be found in terms of the PI values obtained from the main renal arteries. These results have demonstrated the value and importance of the RI value as an indicator of tubulointerstitial damage in chronic renal disease patients with decreased GFR. Unlike many other similar studies, RI values from different renal disease stages were studied and compared in our study, and as a result, it was shown that there was a linear increase in RI values in patients with advancing renal disease. These results clearly indicate the importance of following renal Doppler and RI parameters in patients with clinically and histopathologically proven chronic renal disease, in terms of determination of disease stage and prognosis.

Renal dimensions and parenchymal thicknesses are affected by the height and weight of an individual. On the other hand, diseases such as rapidly progressive glomerulonephritis and amyloid nephropathy lead to an increase in renal dimensions [18]-[20]. Renal dimensions and parenchymal thickness do not seem to be practical parameters for the diagnosis and follow-up of chronic renal disease. In our study too, statistically significant differences were found between the patient and control groups and also between various chronic renal disease stages in terms of kidney dimensions and parenchymal thicknesses, but it still must be emphasized that these are not useful parameters in the determination of chronic renal disease and its stage.

Renal Doppler parameters and renal parenchymal disease stages were comparatively evaluated in this study. But, histopathological staging which directly affects Doppler parameters could not be realized due to the invasiveness and high cost of the biopsy procedure. This seems to be a restrictive factor of our study.

There were two fundamental results of this study:

1) Doppler US must be used routinely in patients with advanced stage renal parenchymal disease. A statistically significant relation was found between the RI values obtained from the interlobar arteries and main renal arteries, and this relation was getting stronger with the increase in disease stage. Any increase in the RI and PI values obtained from the main renal and interlobar arteries must bring to mind the possibility of advancing renal damage and interstitial fibrosis. 2) Groups with and without renal parenchymal damage can be differentiated by means of comparing the total PI values obtained from the right and left main renal and interlobular arteries. This differentiation can be made over a cut-off value of 2.15 , with a sensitivity of $90 \%$ and a specificity of $86.7 \%$.

\section{Conflicts of Interest and Source of Funding}

All contributing authors declare that they have no conflicts of interest and no source of funding.

\section{References}

[1] Omer, M.A.A., Eljack, A.H., Garalnabi, M.E.M., Mahmoud, M.Z., Elseid, M. and Edam, G.A. (2014) Ultrasonographic Characteristics of Diabetes Impacts in Kidneys’ Morphology. Open Journal of Radiology, 4, 301-308. http://dx.doi.org/10.4236/ojrad.2014.44039

[2] Meguid El Nahas, A. and Bello, A.K. (2005) Chronic Kidney Disease: The Global Challenge. Lancet, 365, 331-340. http://dx.doi.org/10.1016/S0140-6736(05)17789-7

[3] Eriksen, B.O. and Ingebretsen, O.C. (2006) The Progression of Chronic Kidney Disease: A 10-Year Population-Based Study of the Effects of Gender and Age. Kidney International, 69, 375-382. http://dx.doi.org/10.1038/sj.ki.5000058

[4] Clark, L.E. and Khan, I. (2010) Outcomes in CKD: What We Know and What We Need to Know. Nephron Clinical Practice, 114, 95-102. http://dx.doi.org/10.1159/000254381

[5] Bigé, N., Lévy, P.P., Callard, P., Faintuch, J.M., Chigot, V., Jousselin, V., et al. (2012) Renal Arterial Resistive Index Is Associated with Severe Histological Changes and Poor Renal Outcome during Chronic Kidney Disease. BMC Nephrology, 13, 139. http://dx.doi.org/10.1186/1471-2369-13-139

[6] Sugiura, T. and Wada, A. (2009) Resistive Index Predicts Renal Prognosis in Chronic Kidney Disease. Health Nephrology Dialysis Transplantation, 24, 2780-2785. http://dx.doi.org/10.1093/ndt/gfp121

[7] Ghadirpour, A., Tarzamni, M.K., Naghavi-Behzad, M., Abedi-Azar, S., Koushavar, H. and Nezami, N. (2014) Renal vascular Doppler Ultrasonographic Indices and Carotid Artery Intima-Media Thickness in Diabetic Nephropathy. Medical Ultrasonography, 16, 95-99. http://dx.doi.org/10.11152/mu.2013.2066.162.ag1mkt2

[8] Viazzi, F., Leoncini, G., Derchi, L.E. and Pontremoli, R. (2014) Ultrasound Doppler Renal Resistive Index: A Useful Tool for the Management of the Hypertensive Patient. Journal of Hypertension, 32, 149-153. http://dx.doi.org/10.1097/HJH.0b013e328365b29c

[9] Stevens, L.A., Coresh, J., Feldman, H.I., Greene, T., Lash, J.P., Nelson, R.G., et al. (2007) Evaluation of the Modifica- 
tion of Diet in Renal Disease Study Equation in a Large Diverse Population. Journal of the American Society of Nephrology, 18, 749-757. http://dx.doi.org/10.1681/ASN.2007020199

[10] Soker, G., Ozturk, A.B., Gulek, B., Kuscu, F., Doğan, U.B. and Yilmaz, C. (2015) Doppler Ultrasonography Helps Discriminate between Cirrhotic and Non-Cirrhotic Patients with Viral B and C Hepatitis. Diagnostic and Interventional Imaging.

[11] Dib, F.R., Duarte, G., Sala, M.M., Ferriani, R.A. and Berezowski, A.T. (2003) Prospective Evaluation of Renal Artery Resistance and Pulsatility Indices in Normal Pregnant Women. Ultrasound in Obstetrics \& Gynecology, 22, 515-519. http://dx.doi.org/10.1002/uog.240

[12] Nath, K.A. (1992) Tubulointerstitial Changes as a Major Determinant in the Progression of Renal Damage. American Journal of Kidney Diseases, 20, 1-17. http://dx.doi.org/10.1016/S0272-6386(12)80312-X

[13] Platt, J.F., Ellis, J.H., Rubin, J.M., DiPietro, M.A. and Sedman, A.B. (1990) Intrarenal Arterial Doppler Sonography in Patients with Nonobstructive Renal Disease: Correlation of Resistive Index with Biopsy Findings. American Journal of Roentgenology, 154, 1223-1227. http://dx.doi.org/10.2214/ajr.154.6.2110732

[14] Ikee, R., Kobayashi, S., Hemmi, N., Imakiire, T., Suzuki, S. and Miura, S. (2005) Correlation between the Resistive Index by Doppler Ultrasound and Kidney Function and Histology. American Journal of Kidney Diseases, 46, 603-609. http://dx.doi.org/10.1053/j.ajkd.2005.06.006

[15] Mostbeck, G.H., Kain, R., Mallek, R., Derfler, K., Walter, R., Havelec, L., et al. (1991) Duplex Doppler Sonography in Renal Parenchymal Disease. Histopathologic Correlation. Journal of Ultrasound in Medicine, 10, 189-194.

[16] Izumi, M., Sugiura, T., Nakamura, H., Nagatoya, K., Imai, E. and Hori, M. (2000) Differential Diagnosis of Prerenal Azotemia from Acute Tubular Necrosis and Prediction of Recovery by Doppler Ultrasound. American Journal of Kidney Diseases, 35, 713-719. http://dx.doi.org/10.1016/S0272-6386(00)70020-5

[17] Sugiura, T., Nakamori, A., Wada, A. and Fukuhara, Y. (2004) Evaluation of Tubulointerstitial Injury by Doppler Ultrasonography in Glomerular Diseases. Clinical Nephrology, 61, 119-126. http://dx.doi.org/10.5414/CNP61119

[18] Hanamura, K., Tojo, A., Kinugasa, S., Asaba, K. and Fujita, T. (2012) The Resistive Index Is a Marker of Renal Function, Pathology, Prognosis, and Responsiveness to Steroid Therapy in Chronic Kidney Disease Patients. International Journal of Nephrology, 2012, Article ID: 139565. http://dx.doi.org/10.1155/2012/139565

[19] Emamian, S.A., Nielsen, M.B., Pedersen, J.F. and Ytte, L. (1993) Kidney Dimensions at Sonography: Correlation with Age, Sex, and Habitus in 665 Adult Volunteers. American Journal of Roentgenology, 160, 83-86. http://dx.doi.org/10.2214/ajr.160.1.8416654

[20] Miletić, D., Fuckar, Z., Sustić, A., Mozetic, V., Stimac, D. and Zauhar, G. (1998) Sonographic Measurement of Absolute and Relative Renal Length in Adults. Journal of Clinical Ultrasound, 26, 185-189. http://dx.doi.org/10.1002/(SICI)1097-0096(199805)26:4<185::AID-JCU1>3.0.CO;2-9 